SHEP 28-10

CP3-Origins-2010-38

\title{
Scaling relations for the entire spectrum in mass-deformed conformal gauge theories
}

\author{
Luigi Del DeBbio $\propto 1$ \& Roman Zwicky 2 \\ a School of Physics and Astronomy, University of Edinburgh, Edinburgh \\ EH9 3JZ, Scotland \\ ${ }^{b}$ School of Physics \& Astronomy, University of Southampton, Highfield, \\ Southampton SO17 1BJ, UK
}

\begin{abstract}
We consider mass-deformed conformal gauge theories (mCGT) and investigate the scaling behaviour of hadronic observables as a function of the fermion mass. Applying renormalization group arguments directly to matrix elements, we find $m_{H} \sim m^{1 /\left(1+\gamma_{*}\right)}$ and $F \sim m^{\eta_{F}\left(\gamma_{*}\right)}$ for given $\eta_{F}\left(\gamma_{*}\right)$, for the hadronic masses and the decay constants respectively, thereby generalizing our results from a previous paper to the entire spectrum. Applying the Hellmann-Feynman theorem to the trace anomaly we obtain the hadron mass scaling independent of renormalization group arguments. From the trace anomaly we obtain a relation reminiscent of the Gell-Mann Oakes Renner relation in QCD. Using the new results we discuss the scaling of the $S$-parameter inside the conformal window. Finally, we discuss how spectral representations can be used to relate the mass and decay constant trajectories.
\end{abstract}

\footnotetext{
${ }^{1}$ luigi.del.debbio@ed.ac.uk

22Roman.Zwicky@soton.ac.uk
} 


\section{Introduction}

The physics of quantum field theories with an infrared fixed point (IRFP) is characterized by scale invariance at large distances, which implies a massless spectrum, and unbroken chiral symmetry. Gauge theories coupled to massless fermions that remain asymptotically free in the ultraviolet and have an IRFP are said to lie in the conformal window. An example of fixed-point, in the weak coupling regime, is due to Banks and Zaks [1]. For lower values of $N_{f}$ the critical coupling is expected to be larger. In supersymmetry, where the nonperturbative dynamics can be studied by analytical methods - see e.g. Ref. [2] for a review - there are examples of strong coupling fixed-points by virtue of electric-magnetic duality.

Identifiying a conformal fixed point at strong coupling in a nonsupersymmetric theory is an interesting theoretical challenge. Techniques have been developed in recent years to understand the phase structure of gauge theories, see e.g. Refs. [3, 4] for recent results and references. In this work we will consider gauge theories minimally coupled to $N_{f}$ Dirac fermions in arbitrary representations of the gauge group. All fermions are degenerate, even though different fermion masses can be easily accomodated. The fermionic mass term is a relevant deformation that drives the theory away from conformality. We refer to these theories as mass-deformed conformal gauge theories (mCGT). Mass deformed theories develop a string tension and thus lead to the formation of massive bound states which shall call hadrons. 5, 6] It is the scaling of the hadronic spectrum and the decay constants as a function of the fermion mass and its applications that we want to consider in this study.

Besides their theoretical interest, conformal theories deformed away from the fixed point are interesting candidates technicolor [7, 8, 9] model building. The important issue for phenomenology is the characterization of the fixed point, through the computation of the relevant critical exponents. The latter are relevant for technicolor searches at colliders [11, 12].

The lattice formulation of gauge theories provides a powerful tool to investigate the nonperturbative dynamics from first principles. Monte Carlo simulations are necessarily performed at finite fermion mass, the signature of an IRFP can be found by studying the scaling of physical quantities with the fermion mass as proposed in Refs. [13]. The scaling laws are dictated by the critical exponents of the fixed point, and have been studied intensively in recent numerical investigations [6, 14, 15].

In this work we: 
- provide scaling laws for condensates, hadron masses, decay constants for the entire spectrum.

- derive the scaling law for the hadron masses from the trace anomaly and the Hellmann-Feynman theorem without using RG arguments. The relation obtained from the trace anomaly is reminiscent of the GMOR relation in QCD.

- reiterate on scaling laws for the S-parameter using our results.

- give the relation between the mass and decay constant trajectories

- suggest to probe the beta function via the trace anomaly and provide a presentation of the beta function which resembles the NSVZ betafunction [22].

In connection with the first item it is worthwhile to note out that in a previous work [10, we have derived the scaling of the hadron masses and decay constants, for the lowest-lying state in any channel with given quantum

numbers, using renormalization group ( $R G$ ) arguments on correlators for large Euclidian times.

\section{Mass scaling from anomalous dimensions}

Consider an operator $\mathcal{O}(x ; \mu)$ renormalized at the scale $\mu$, with scaling dimension $\Delta_{\mathcal{O}}$ and quantum numbers such that the matrix element

$$
T_{\varphi_{1} \mathcal{O} \varphi_{2}}(g, \hat{m}, \mu) \equiv\left\langle\varphi_{2}|\mathcal{O}(0)| \varphi_{1}\right\rangle, \quad \Delta_{\mathcal{O}}=d_{\mathcal{O}}+\gamma_{\mathcal{O}}
$$

is non-vanishing. The symbols $d_{\mathcal{O}}$ and $\gamma_{\mathcal{O}}$ denote the engineering and anomalous dimension respectively. We have indicated the dependence on the two renormalized parameters of the mCGT - the rescaled mass $\hat{m} \mu=m$, and the gauge coupling $g$ - as well as on the scale $\mu$. The kets $\left|\varphi_{i}\right\rangle$, for $i=1,2$, denote physical states with scaling dimensions $\Delta_{\varphi_{i}}=d_{\varphi_{i}}$.

We closely follow part of the argumentation in our previous paper [10], where we applied renormalization group (RG) arguments to study the scaling of field correlators; here we focus on matrix elements of the type introduced in Eq. (11), adding to the discussion the observation that the physical states 
are free of anomalous dimensions (i.e. $\Delta_{\varphi_{i}}=d_{\varphi_{i}}$ ). Close to the fixed point the $\mathrm{RG}$ transformation $\mu=b \mu^{\prime}$ on the matrix element results in:

$$
T_{\varphi_{1} \mathcal{O} \varphi_{2}}(g, \hat{m}, \mu)=b^{-\gamma_{\mathcal{O}}} T_{\varphi_{1} \mathcal{O} \varphi_{2}}\left(g^{\prime}, \hat{m}^{\prime}, \mu^{\prime}\right) .
$$

Near a non-trivial fixed point the couplings display powerlike behaviour:

$$
g^{\prime}=b^{y_{g}} g, \quad \hat{m}^{\prime}=b^{y_{m}} \hat{m},
$$

with

$$
y_{m}=1+\gamma_{*},
$$

where $\gamma_{*}$ denotes the anomalous dimension of the mass at the fixed point. Neglecting the irrelevant coupling $g\left(y_{g}<0\right) \sqrt{3}$ and multiplying all mass units by the factor $b$ we obtain:

$$
T_{\varphi_{1} \mathcal{O} \varphi_{2}}\left(\hat{m}^{\prime}, \mu^{\prime}\right)=b^{-\left(d_{\mathcal{O}}+d_{\varphi_{1}}+d_{\varphi_{2}}\right)} T_{\varphi_{1} \mathcal{O} \varphi_{2}}\left(\hat{m}^{\prime}, \mu\right),
$$

Choosing $b$ such that $\hat{m}^{\prime}=1$, combining equations (2) and (5) yields:

$$
T_{\varphi_{1} \mathcal{O} \varphi_{2}}(\hat{m}, \mu) \sim(\hat{m})^{\left(\Delta_{\mathcal{O}}+d_{\varphi_{1}}+d_{\varphi_{2}}\right) / y_{m}} .
$$

Note that in the approximation made there are no higher order corrections. Such correction originate from taking into account corrections to the beta function of the form $\beta=A m^{\eta_{\beta}}$ with $\eta_{\beta}>0$.

The result in Eq. (6) can also be derived starting from correlation functions. For example for the matrix element the operator $\mathcal{O}$ between one physical state and the vacuum we have:

$$
\begin{aligned}
& \left(p_{1}^{2}-m_{\varphi_{1}}^{2}\right) i \int d^{4} x e^{i p_{1} \cdot x}\left\langle 0\left|T \mathcal{O}(0) \Phi_{1}(x)\right| 0\right\rangle= \\
& \quad=\left(p_{1}^{2}-m_{\varphi_{1}}^{2}\right)\left(\frac{\left\langle 0|\mathcal{O}| \varphi_{1}\right\rangle\left\langle\varphi_{1}\left|\Phi_{1}\right| 0\right\rangle}{p_{1}^{2}-m_{\varphi_{1}}^{2}}+\ldots\right),
\end{aligned}
$$

where $\Phi_{1}$ denotes an interpolating operator for the state $\varphi_{1}$. All other contributions but the $\varphi_{1}$-pole vanish for on-shell momenta. Generalization to the case of two physical states is straightforward.

The scaling relations for the decay constants given in Table 1 of Ref. [10] can be readily rederived from the considerations presented above, borrowing the relation $m_{H} \sim m^{1 /\left(1+\gamma_{*}\right)}$ from the next section. They apply trivially to all one-particle stable states in the spectrum and not only to the lowest lying state as in Ref. [10].

\footnotetext{
${ }^{3}$ We remind the reader that the assumption is, to be verified by lattice simulations,
} 


\begin{tabular}{l|l|l|l|l|r}
$\mathcal{O}$ & $\operatorname{def}$ & $\left\langle 0|\mathcal{O}| J^{\mathrm{P}(\mathrm{C})}(p)\right\rangle$ & $J^{\mathrm{P}(\mathrm{C})}$ & $\Delta_{\mathcal{O}}$ & $\eta_{G[F]}$ \\
\hline$S$ & $\bar{q} q$ & $G_{S}$ & $0^{++}$ & $3-\gamma_{*}$ & $\left(2-\gamma_{*}\right) / y_{m}$ \\
$S^{a}$ & $\bar{q} \lambda^{a} q$ & $G_{S^{a}}$ & $0^{+}$ & $3-\gamma_{*}$ & $\left(2-\gamma_{*}\right) / y_{m}$ \\
$P^{a}$ & $\bar{q} i \gamma_{5} q$ & $G_{P^{a}}$ & $0^{-}$ & $3-\gamma_{*}$ & $\left(2-\gamma_{*}\right) / y_{m}$ \\
$V$ & $\bar{q} \gamma_{\mu} q$ & $\epsilon_{\mu}(p) M_{V} F_{V}$ & $1^{--}$ & 3 & $1 / y_{m}$ \\
$V^{a}$ & $\bar{q} \gamma_{\mu} \lambda^{a} q$ & $\epsilon_{\mu}(p) M_{V} F_{V^{a}}$ & $1^{-}$ & 3 & $1 / y_{m}$ \\
$A^{a}$ & $\bar{q} \gamma_{\mu} \gamma_{5} \lambda^{a} q$ & $\epsilon_{\mu}(p) M_{A} F_{A^{a}}$ & $1^{+}$ & 3 & $1 / y_{m}$ \\
& & $i p_{\mu} F_{P^{a}}$ & $0^{-}$ & 3 & $1 / y_{m}$
\end{tabular}

Table 1: Scaling laws, $G[F] \sim m^{\eta_{G[F]}}$ for decay constants. The symbol $y_{m} \equiv 1+\gamma_{*}$ denotes the scaling dimension of the mass and $\Delta_{\mathcal{O}}=d_{\mathcal{O}}+\gamma_{\mathcal{O}}$. The symbol $a$ denotes the adjoint flavour index, and $\lambda^{a}$ are the generators normalized as $\operatorname{tr}\left[\lambda^{a} \lambda^{b}\right]=2 \delta^{a b}$. No such simple expression exists for the axial singlet current because of the chiral anomaly [10].

\subsection{Scaling of hadron masses}

The approach that we have introduced in the previous section, namely the study of matrix elements of given operators between physical states, can also be used to investigate the scaling of the hadron masses with the fermion mass.

RG arguments applied to two-point functions in Refs. [10] 6 ] led to the scaling relation:

$$
M_{H} \sim m^{1 / y_{m}}
$$

for the lowest state in any given channel. Here we shall generalize it to the entire spectrum using the trace or scale anomaly of the energy momentum tensor.

Let us first state two general facts. When considering its matrix elements between on-shell (i.e. physical) states, the trace of the energy momentum

that we are studying the theory in a neighbourhood of a fixed point where the mass is the only relevant coupling.

4 This relation was first proposed in Ref. [5] by looking at the pole mass around the Banks-Zaks type fixed-point. 
tensor assumes the following form [17]:

$$
\left.\theta_{\alpha}{ }^{\alpha}\right|_{\text {on-shell }}=\frac{1}{2 g} \beta G^{2}+N_{f} m\left(1+\gamma_{m}\right) \bar{q} q
$$

where $\beta=\frac{\partial g}{d \ln \mu}$. On the other hand the matrix element of the energy momentum tensor between two physical states can be written as, e.g. [16],

$$
\left\langle H(p)\left|\theta_{\alpha \delta}\right| H(p)\right\rangle=2 p_{\alpha} p_{\delta}
$$

which is consistent with the relativistic normalization $\langle H(\vec{p}) \mid H(\vec{k})\rangle=2 E_{p} \delta^{(3)}(\vec{p}-$ $\vec{k})$. Note that all operators appearing in eq. (10) are intended to be renormalized at some scale $\mu$.

Taking the trace of Eq. (10), equating with (9), neglecting the $\beta$ function, and adopting our notation $\gamma_{m}=\gamma_{*}$ yields

$$
2 M_{H}^{2}=N_{f}\left(1+\gamma_{*}\right) m\langle H|\bar{q} q| H\rangle
$$

a relation reminiscent of the Gell-Mann Oakes Renner relation in QCD. The scaling of the hadron masses, for the entire spectrum, can be obtained in two alternative ways.

1. From (6) , with $d_{H(p)}=-1$ and $\Delta_{m \bar{q} q}=4$, it follows that $m\langle H|\bar{q} q| H\rangle \sim$ $m^{2 / y_{m}}$ and thus Eq. (11) implies (8). For the scaling corrections, the same remarks apply as for the quantity in Eq. (6).

2. The Feynman-Hellmann theorem states that,

$$
\frac{\partial E_{\lambda}}{\partial \lambda}=\left\langle\psi(\lambda)\left|\frac{\partial \hat{H}(\lambda)}{\partial \lambda}\right| \psi(\lambda)\right\rangle
$$

the variation of the energy with respect to a parameter equals the expectation value of the variation of the Hamiltonian. In our case, we consider the derivative with respect to the mass $m$. Taking into account our chosen normalization of states, Eq. (12) yields

$$
m \frac{\partial M_{H}^{2}}{\partial m}=N_{f} m\langle H|\bar{q} q| H\rangle .
$$

\footnotetext{
${ }^{5}$ When evaluated on states, in this notation, it is understood that only the connected part is evaluated.
} 
Equating with Eq. (11) we get

$$
m \frac{\partial M_{H}}{\partial m}=\frac{1}{1+\gamma_{*}} M_{H}
$$

which implies Eq. (8) with (44). It is worth emphasizing that this derivation does not depend on RG arguments.

Note that the same statement applies to the decay rates $\Gamma_{A \rightarrow B C . .} \sim m^{1 / y_{m}}$ since the latter transition Hamiltonian is free from anomalous scaling. Similar arguments for the scaling of decay rates were already discussed in our previous paper [10].

\subsection{A remark on the $\beta$-function}

Finally, Eqs. (9) and (10) imply a relation between the beta function and the mass anomalous dimension:

$$
\beta=\frac{A_{H}+\gamma_{m} B_{H}}{G_{H}},
$$

where

$$
\begin{aligned}
A_{H} & =2 M_{H}^{2}-m N_{f}\langle H|\bar{q} q| H\rangle, \\
B_{H} & =m N_{f}\langle H|\bar{q} q| H\rangle, \\
G_{H} & =\left\langle H\left|G^{2}\right| H\right\rangle .
\end{aligned}
$$

The detailed investigation of this relation is postponed to further studies [21]. The connection with the so-called NSVZ beta function [22] is obvious. This relation could, for instance, be used to probe the $\beta$-function in mCGT. Note that the combinations $A_{H} / G_{H}$ and $B_{H} / G_{H}$ are independent of the state $H$.

\subsection{Remarks on S-parameter}

The determination of the S-parameter for gauge theories is important for technicolor model building, be it for walking-like or conformal behaviour. In this section, we discuss the implications of our scaling relations for the

S-parameter. These analytical results for the scaling of the S-parameter lead to new criteria that can help to distinguish a conformal phase from a walking phase. 
Using the results valid for the entire spectrum rather than the lowest lying state, we can put the remarks on the $S$ parameter of mCGT that were presented in the conclusions of Ref. [10] on more solid grounds.

Defining the dimensionless correlation function $\Pi_{V-A}\left(q^{2}\right)$ from the correlation function:

$$
\begin{aligned}
\Pi(q)_{a b}^{\mu \nu} & \equiv i \int d^{4} x e^{i q \cdot x}\left\langle 0\left|T\left(V_{a}^{\mu}(x) V_{b}^{\nu}(0)-(V \leftrightarrow A)\right)\right| 0\right\rangle \\
& =\left(-q^{2} g^{\mu \nu}+q^{\mu} q^{\nu}\right) \delta_{a b} \Pi_{V-A}\left(q^{2}\right)+g_{\mu \nu} m^{2} \Pi_{A}\left(q^{2}\right) .
\end{aligned}
$$

Saturating the correlation function with hadronic states, one would expect

$$
\Pi_{V-A}\left(q^{2}\right)=\frac{f_{V}^{2}}{m_{V}^{2}-q^{2}}-\frac{f_{A}^{2}}{m_{A}^{2}-q^{2}}-\frac{f_{P}^{2}}{m_{P}^{2}-q^{2}}+\ldots .
$$

As discussed in Ref. [18, the scaling of the correlator with the fermion mass depends on the order in which the limits $q^{2} \rightarrow 0$ and $m \rightarrow 0$ are taken. When $q^{2} \rightarrow 0$ at fixed $m$, the scaling laws for the hadronic masses and decay constants imply that

$$
\Pi_{V-A}(0) \sim O\left(m^{0}\right)
$$

our analysis only yields the scaling law, but no information on the prefactor. The analysis presented in Refs. [18, 19, 20] actually aims at a lower bound for the prefactor. We would like to add that we cannot exclude that the vector and axial contributions conspire to cancel up some order in $m$. For the case where we take the limit $m \rightarrow 0$ at fixed $q^{2}$, we obtain:

$$
\Pi_{V-A}\left(q^{2}\right) \stackrel{-q^{2} \gg\left(\Lambda_{U}\right)^{2}}{\sim} \frac{m^{2 / y_{m}}}{q^{2}}+O\left(\frac{m^{2}}{q^{2}}\right),
$$

with the same caveat we highlighted for Eq. (18). The condition $-q^{2} \gg$ $\left(\Lambda_{\mathrm{U}}\right)^{2}$, where $\Lambda_{\mathrm{U}}$ is the scale where asymptotic freedom sets in, as otherwise the highest resonances $M_{\text {high }}^{2}>q^{2}$ and this would imply a behaviour as in Eq. (18). Note that for $-q^{2} \gg\left(\Lambda_{\mathrm{U}}\right)^{2}$ the correlator $\Pi_{V-A}\left(q^{2}\right)$ does still receive perturbative correction of the order $\mathrm{m}^{2} / q^{2}$ due to the explicit breaking of chiral symmetry. These corrections can already be seen in a perturbative computation near the Banks-Zaks fixed point, as was pointed out in Ref. [18]. As emphasized in Ref. [10] the behaviour (18) is distinct from the correlator of a gauge theory with broken chiral symmetry has a pion pole and thus behaves like $\Pi(0) \sim O\left(m^{-1}\right)$. This limit is useful to identify a conformal behaviour in a lattice simulation, where the limit $m \rightarrow 0$ at fixed $q^{2}$ can be investigated [25, 23, 24]. 


\section{Relating the mass and decay constant tra- jectory}

We have established the scaling of hadronic masses and decay constants in terms of the mass. The nature of the spectrum remains an open question, but we we can say something about the relation between the masses and the decay constants in the large- $N_{c}$ limit. In the latter limit the width it is believed to be $N_{c}$ using the same $N_{c}$ scaling arguments as in QCD. A two-point function, of an operator $\mathcal{O}$ coupling to states $\left|H_{n}\right\rangle$, assumes the following form:

$$
\Delta\left(q^{2}\right) \sim \int_{x} e^{i x q}\langle 0|\mathcal{O}(x) \mathcal{O}(0)| 0\rangle=\sum_{n} \frac{\left|g_{H_{n}}\right|^{2}}{q^{2}+M_{H_{n}}^{2}},
$$

in Euclidean space. The symbol $g_{H_{n}} \equiv\left\langle 0|\mathcal{O}| H_{n}\right\rangle$ defines what we call decay constant in this context.

On the other hand when the mass is sent to zero, the spectral representation for the two point function combined with scale invariance implies [27]:

$$
\Delta\left(q^{2}\right)=\int_{0}^{\infty} \frac{d s s^{1-\gamma_{*}}}{q^{2}+s}+\text { s.t } \sim\left(q^{2}\right)^{1-\gamma_{*}},
$$

where we have implicitly parametrized the scaling dimension of $\mathcal{O}$ as $\Delta_{\mathcal{O}}=$ $3-\gamma_{*}$. The symbol s.t. stands for ultraviolet subtraction terms which are not relevant for our argument. Thus the masses and decay constants have to behave in such a way as to reproduce (21). It is readily seen that this is achieved as follows:

$$
M_{H_{n}}^{2} \sim \alpha_{n} m^{\frac{2}{1+\gamma_{*}}}, \quad g_{H_{n}}^{2} \sim \alpha_{n}^{\prime}\left(\alpha_{n}\right)^{1-\gamma_{*}} m^{\frac{2\left(2-\gamma_{*}\right)}{1+\gamma_{*}}}
$$

The symbol $\alpha_{n}$ denotes any monotonic increasing function and $\alpha_{n}^{\prime}$ is the derivative w.r.t. to $n$. The freedom of choosing $\alpha_{n}$ corresponds to the freedom of changing variables in the integral representation (21) 6 .

\footnotetext{
${ }^{6}$ Similar considerations can also be applied to large- $N_{c}$ QCD. In the latter case a linear $\alpha_{n} \sim n$ (Regge trajectory) spectrum is expected. In the case where $\mathcal{O} \rightarrow \bar{q} \gamma_{\mu} q$ and at large momentum transfer $\Delta_{\mu \nu} \sim\left(q^{2} g_{\mu \nu}-q_{\mu} q_{\nu}\right) \ln \left(q^{2}\right)$ which is easily verified by perturbation theory justified by asymptotic freedom. This suggests $\left.\gamma^{*}\right|_{\text {eff }}=1$ and $g_{n} \sim O\left(n^{0}\right)$. See e.g. Ref. [26] where this type of model has been suggested. N.B. the role of the parameter $m$ is played by $\Lambda_{\mathrm{QCD}}$ and the powers are simply the engineering dimensions of the mass and decay constants.

${ }^{7}$ Our discussion formally resembles the deconstruction of a scale invariant spectrum in
} 
Acknowledgements: LDD and RZ acknowledge the support of advanced STFC fellowships. We are grateful to John Donoghue for correspondance and Nick Evans \& Tim Morris for discussions.

\section{References}

[1] T. Banks and A. Zaks, Nucl. Phys. B 196, 189 (1982).

[2] K. A. Intriligator and N. Seiberg, Nucl. Phys. Proc. Suppl. 45BC, 1 (1996) arXiv:hep-th/9509066.

[3] F. Sannino, Acta Phys. Polon. B 40, 3533 (2009) arXiv:0911.0931 [hep-ph]].

[4] E. Poppitz and M. Unsal, JHEP 0909, 050 (2009) arXiv:0906.5156 [hep-th]].

[5] V. A. Miransky, Phys. Rev. D59 (1999) 105003. [hep-ph/9812350].

[6] L. Del Debbio, B. Lucini, A. Patella, C. Pica and A. Rago, Phys. Rev. D 80, 074507 (2009) arXiv:0907.3896 [hep-lat]].

[7] F. Sannino and K. Tuominen, Phys. Rev. D 71, 051901 (2005) arXiv:hep-ph/0405209.

[8] M. A. Luty and T. Okui, JHEP 0609, 070 (2006) arXiv:hep-ph/0409274.

[9] F. Sannino, R. Zwicky, Phys. Rev. D79 (2009) 015016. arXiv:0810.2686 [hep-ph]].

[10] L. Del Debbio and R. Zwicky, Phys. Rev. D 82 (2010) 014502 arXiv:1005.2371 [hep-ph]].

[11] J. Galloway, J. A. Evans, M. A. Luty and R. A. Tacchi, arXiv:1001.1361 [hep-ph].

27. The difference is that we give a specific interpretation of the spacing of the resonances whereas in 27] the spacing is introduced as a mathematical tool. 
[12] H. S. Fukano and F. Sannino, Phys. Rev. D 82 (2010) 035021 arXiv:1005.3340 [hep-ph]].

R. S. Chivukula and E. H. Simmons, Phys. Rev. D 82, 033014 (2010) arXiv:1005.5727 [hep-lat]].

[13] M. A. Luty, JHEP 0904, 050 (2009) [arXiv:0806.1235 [hep-ph]].

[14] T. DeGrand, Phys. Rev. D 80, 114507 (2009) arXiv:0910.3072 [heplat]].

[15] L. Del Debbio, B. Lucini, A. Patella, C. Pica and A. Rago, Phys. Rev. D 82, 014509 (2010) [arXiv:1004.3197 [hep-lat]].

[16] J. F. Donoghue, E. Golowich and B. R. Holstein, Camb. Monogr. Part. Phys. Nucl. Phys. Cosmol. 2 (1992) 1.

[17] S. L. Adler, J. C. Collins and A. Duncan, Phys. Rev. D 15 (1977) 1712. J. C. Collins, A. Duncan and S. D. Joglekar, Phys. Rev. D 16 (1977) 438.

N. K. Nielsen, Nucl. Phys. B 120 (1977) 212.

P. Minkowski, PRINT-76-0813-BERN

K. Fujikawa, Phys. Rev. D 23 (1981) 2262.

[18] F. Sannino, arXiv:1006.0207 [hep-lat].

[19] F. Sannino, arXiv:1007.0254 [hep-ph].

[20] S. Di Chiara, C. Pica and F. Sannino, arXiv:1008.1267 [hep-ph].

[21] L. Del Debbio and R. Zwicky, in preparation

[22] V. A. Novikov, M. A. Shifman, A. I. Vainshtein and V. I. Zakharov, Nucl. Phys. B 229 (1983) 381. V. A. Novikov, M. A. Shifman, A. I. Vainshtein and V. I. Zakharov, Phys. Lett. B 166 (1986) 329 [Sov. J. Nucl. Phys. 43 (1986) 294] [Yad. Fiz. 43 (1986) 459].

[23] P. A. Boyle, L. Del Debbio, J. Wennekers and J. M. Zanotti [RBC Collaborations and UKQCD Collaborations], Phys. Rev. D 81, 014504 (2010) [arXiv:0909.4931 [hep-lat]]. 
[24] T. DeGrand, arXiv:1006.3777 [hep-lat].

[25] E. Shintani et al. [JLQCD Collaboration], Phys. Rev. Lett. 101, 242001 (2008) arXiv:0806.4222 [hep-lat]].

[26] M. A. Shifman, arXiv:hep-ph/0009131.

[27] M. A. Stephanov, Phys. Rev. D 76, 035008 (2007) arXiv:0705.3049 [hep-ph]]. 